\title{
Tousled-like kinase mediated a new type of cell death pathway in Drosophila
}

\author{
Y Zhang ${ }^{1,2,3}, \mathrm{R}$ Cai ${ }^{1,2,3}, \mathrm{R} \mathrm{Zhou}^{2}, \mathrm{Y} \mathrm{Li}{ }^{*, 2}$ and L Liü,
}

Programmed cell death (PCD) has an important role in sculpting organisms during development. However, much remains to be learned about the molecular mechanism of PCD. We found that ectopic expression of tousled-like kinase (t/k) in Drosophila initiated a new type of cell death. Furthermore, the TLK-induced cell death is likely to be independent of the canonical caspase pathway and other known caspase-independent pathways. Genetically, atg2 RNAi could rescue the TLK-induced cell death, and this function of atg2 was likely distinct from its role in autophagy. In the developing retina, loss of tlk resulted in reduced PCD in the interommatidial cells (IOCs). Similarly, an increased number of IOCs was present in the atg2 deletion mutant clones. However, double knockdown of t/k and atg2 by RNAi did not have a synergistic effect. These results suggested that ATG2 may function downstream of TLK. In addition to a role in development, t/k and atg2 RNAi could rescue calcium overload-induced cell death. Together, our results suggest that TLK mediates a new type of cell death pathway that occurs in both development and calcium cytotoxicity.

Cell Death and Differentiation (2016) 23, 146-157; do:10.1038/cdd.2015.77; published online 19 June 2015

Cell death subtypes can be classified into uncontrollable accidental cell death and regulated cell death (RCD). As a further subtype of RCD, the cell death that occurs in development is referred as programmed cell death (PCD). ${ }^{1}$ Although caspase-dependent apoptosis has crucial roles in development, other type(s) of PCD may exist. ${ }^{2}$ The Drosophila eye is an elegant model system with which to study PCD in development; ${ }^{3,4}$ the patterning of the Drosophila eye is highly stereotypic and well characterized. The development of the fly retina begins in the eye disc of the third instar larvae, where the formation of ommatidium initiates from the differentiation of eight photoreceptor cells ( $R$ cells) followed by the recruitment of four cone cells. At the pupal stage, two primary pigment cells are recruited to surround the cone cells. Then, the interommatidial cells (IOCs) are chosen from a pool of undifferentiated cells and further refined into a highly stereotypical hexagonal lattice. ${ }^{3}$ Each hexagonal lattice contains 12 IOCs, including six secondary pigment cells at the edges, three tertiary pigment cells and three bristle cells at the vertices. 5,6 The undetermined IOCs are then removed by apoptosis. $^{7}$

It has been shown that intercellular communication has an essential role in regulating $\mathrm{IOC}$ apoptosis. ${ }^{8}$ The cone cells and primary pigment cells release survival ligands, such as Spitz, to promote the survival of IOCs, whereas IOCs release Delta to promote the death of their neighbors by activating the Notch pathway. $^{2,8}$ Excessive IOCs are not the only cell type to be eliminated; the perimeter ommatidia are also trimmed during development. This process is mediated by the secretion of a glycoprotein, Wingless, which promotes its own expression in the periphery of the eye and activates the caspase-dependent apoptosis pathway. ${ }^{6}$ The entire cell population of the perimeter ommatidia is eliminated, including the photoreceptor cells, cone cells, primary pigment cells and IOCs. ${ }^{6}$

Apoptosis is an important variant of PCD and is executed by caspases. ${ }^{1}$ In Drosophila, these caspases include Dronc, a main initiator caspase, and DrICE and Dcp-1, which are activated by Dronc. ${ }^{9}$ The activities of caspases can be inhibited by two endogenous proteins, DIAP1 and DIAP2. ${ }^{10}$ Similarly, p35, a viral protein, is capable of inhibiting the caspase activity of DrICE and Dcp-1, resulting in the survival of extra IOCs and primary pigment cells in the adult fly retina. $^{4,9}$ In Drosophila, the RHG (Reaper, Hid and Grim) family members are antagonists of DIAPs. ${ }^{10}$ In cells that are doomed to die, transcription of the RHG genes is increased. ${ }^{11-13}$ In addition, Drosophila p53 can promote apoptosis by acting together with the JNK signaling pathway to regulate the $\mathrm{RHG}$ proapoptotic machinery. ${ }^{14,15}$ Although deletion of the RHG genes blocks the majority of apoptosis, other PCD pathways likely exist during Drosophila eye development. $^{2}$

In addition to apoptosis, other cell death pathways exist, although their roles in eye development are unclear. Ectopic expression of eiger (the fly homolog of mammalian TNF-a) induces cell death in the fly eyes. This type of apoptosis can be weakly inhibited by $\mathrm{p} 35$, but is strongly suppressed by the loss

\footnotetext{
${ }^{1}$ State Key Laboratory of Membrane Biology, School of Life Sciences, Peking University, Beijing, China and ${ }^{2}$ Center of Stroke, Beijing Institute for Brain Disorder and Xuanwu Hospital, Capital Medical University, Beijing, China

*Corresponding author: Y Li or L Liu, Center of Stroke, Beijing Institute for Brain Disorder and Xuanwu Hospital, Capital Medical University, Beijing 100069, China. Tel: +86 10 62759691; Fax: +86 10 62756100; E-mail: yuhongli@pku.edu.cn or leiliu@ccmu.edu.cn

${ }^{3}$ These authors contributed equally to this work.

Abbreviations: TLK, tousled-like kinase; PCD, programmed cell death; IOCs, interommatidial cells; RCD, regulated cell death; RHG, reaper, hid and grim; AIF, apoptosisinducing factor; JNK, Jun N-terminal kinase; MF, morphogenetic furrow; AO, acridine orange; APF, after pupa formation; pH3, anti-phospho-histone H3; TUNEL, TdT-mediated dUTP nick end labeling; PI, propidium iodide

Received 30.6.14; revised 21.4.15; accepted 05.5.15; Edited by E Baehrecke; published online 19.6 .15
} 
of JNK (Jun N-terminal kinase also called BSK) signaling, indicating that the Eiger/JNK-induced RCD is caspaseindependent. ${ }^{16,17}$ Moreover, Drosophila AIF (apoptosisinducing factor)-mediated cell death is also independent of the canonical caspase pathway. ${ }^{18}$ Autophagic cell death has been described to participate in Drosophila embryogenesis and is involved in the removal of the salivary gland and midgut tissues during Drosophila metamorphosis. ${ }^{19-21}$

Beyond development, cell death has important roles in human diseases. ${ }^{22}$ For example, calcium overload is a pivotal stressor that induces cell death in many human diseases, such as stroke, traumatic brain injury, epilepsy, Alzheimer's disease and glaucoma. ${ }^{23-25}$ However, much remains to be learned regarding calcium-induced cell death pathways. ${ }^{26}$

Here, we reported the discovery of a new type of TLKinduced PCD in Drosophila and delineated the function of TLK in both eye development and calcium-induced cell death.

\section{Results}

Overexpression of $t / k$ induced cell death in Drosophila eyes. The adult Drosophila can survive without eyes. ${ }^{27}$ Therefore, a genetic screen with the eye-specific promoter
GMR-Gal4 ${ }^{28}$ and various UAS lines is an elegant approach to discover the function of genes that cause Drosophila lethality. Here, the Gal4/UAS binary expression system is simplified as ' $>$ ' throughout the text. We observed that the overexpression of $t / k$ in the fly eyes (GMR>t/k) resulted in loss of pigmentation (Figure $1 \mathrm{~A}$ a and b). To visualize each ommatidium, a membrane-tagged GFP transgene was added to the $G M R>t / k$ flies (GMR $>m C D 8-G F P / t / k)$. This fly also showed the defective eye phenotype (Figure $1 \mathrm{~A} \mathrm{c}$ ). To further characterize the cell death, we cross-sectioned the eyes to visualize the internal structure. In the GMR-Gal4 control flies, each ommatidium displays a hexagonal profile with $7 \mathrm{R}$ cells and accessory pigment cells ${ }^{29}$ (Figure $1 \mathrm{~A} d$ ). In contrast, almost no intact ommatidia were visible and massive vacuoles were present throughout the eyes of $G M R>t / k$ and $G M R>m C D 8-G F P / t / k$ flies (Figure $1 \mathrm{~A}$ e and f). The defects could be suppressed by two independent $t / k R N A$ lines (Figure 1B). These RNAi lines target different regions of the $t / k$ transcript and are designed to avoid off-target effect. ${ }^{30}$ The quantitative RT-PCR verified that these RNAi lines indeed reduced the tlk transcripts (Supplementary Figure S1).

The absence of ommatidia in GMR $>$ tlk may result from a failure to differentiate or enhanced cell death. To distinguish
A
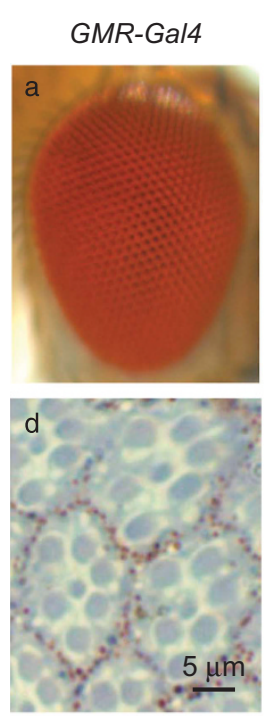

C

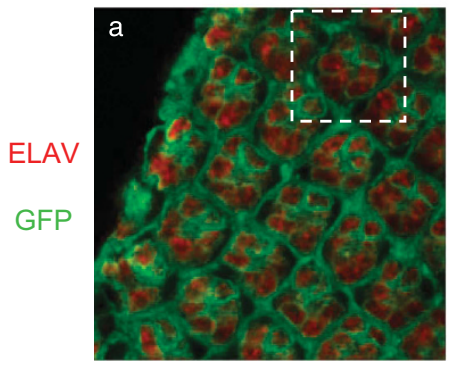

GMR-Gal4/UAS-mCD8GFP; UAS-tlk/+
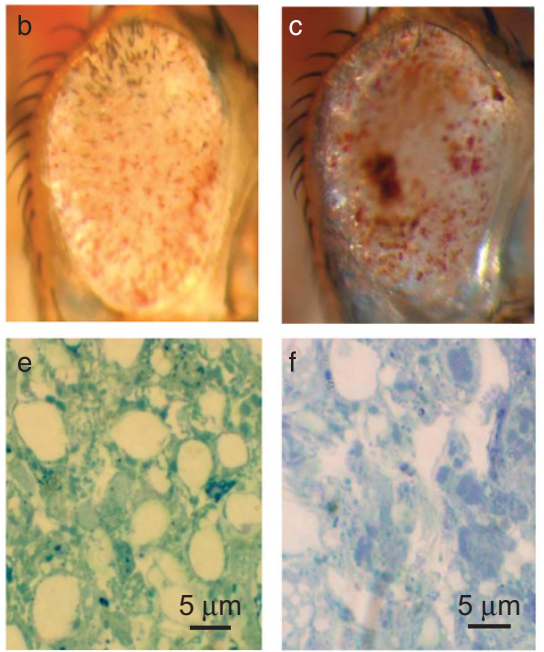

B

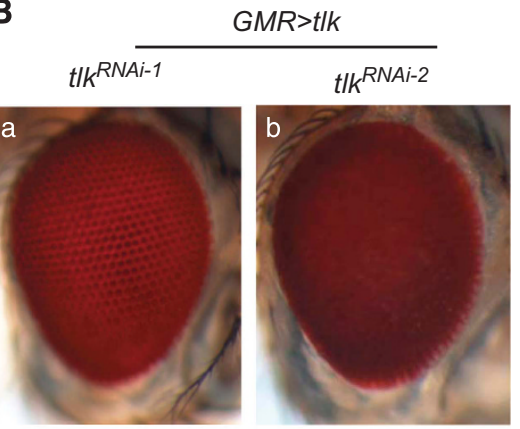

GMR-Gal4/UAS-mCD8-GFP; UAS-tlk/+

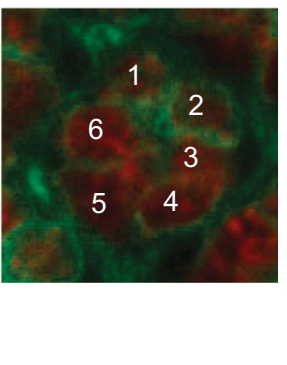

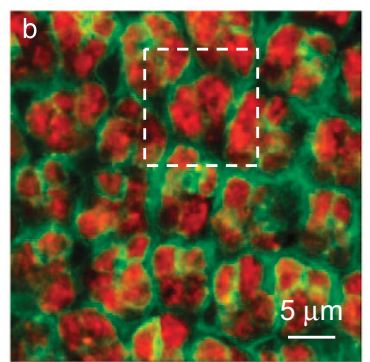

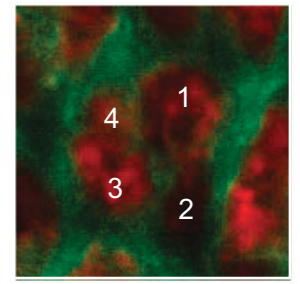

Figure 1 Ectopic expression of tlk induced cell death in Drosophila eyes. (A) Overexpression of tlk induces eye defect. a, b and c are the images of the light microscope. d, e and $f$ shows the toluidine blue-stained semi-thin sections of the eyes. The genotypes are shown on top of each micrograph. (B) The effect of two independent $t / k^{R N A i}$ lines on the GMR > tlk flies. (C) Immunostaining of the eye disc from the 3rd instar larvae. The mCD8-GFP is a membrane-targeted GFP, and ELAV is a neuron marker. The enlarged images show that a cell cluster in $\mathrm{b}$ contains less $\mathrm{R}$ cells than $\mathrm{a}$ 
A

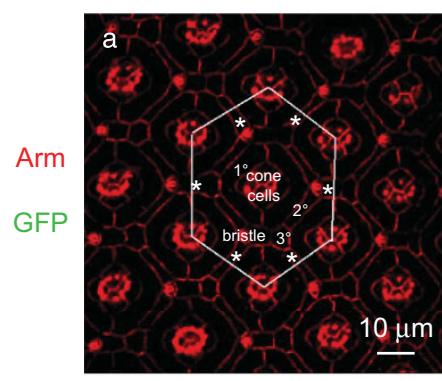

$t / k^{\prime(1) G 0054}$, FRT19A/ey-FLP, Ubi-GFP, FRT19A
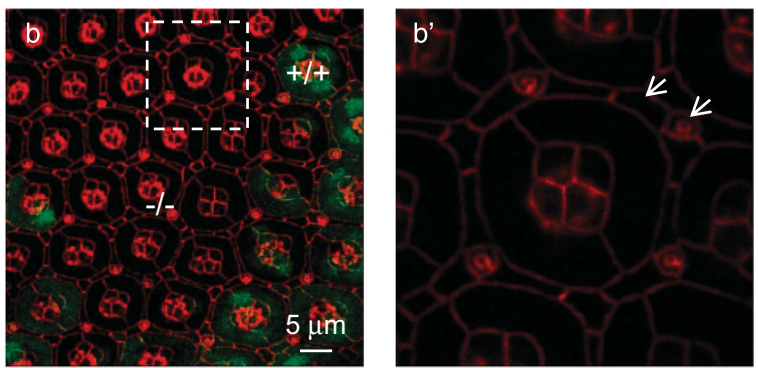

B

\begin{tabular}{lll}
\hline Genotype & $\begin{array}{l}\text { Cells / hexagon } \\
\pm \text { s.e.m. }\end{array}$ & $\begin{array}{l}\triangle \mathrm{IOC} / \text { ommatidium } \\
\pm \text { s.e.m. }\end{array}$ \\
\hline$w^{1118}$ & $21 \pm 0$ & $0 \pm 0$ \\
\hline$t^{\prime(1) \text { G0054 FRT19A/ey- }}$ & $23.38 \pm 0.98^{* *}$ & $0.79 \pm 0.32$ \\
FLP, Ubi-GFP, FRT19A & & \\
\hline
\end{tabular}

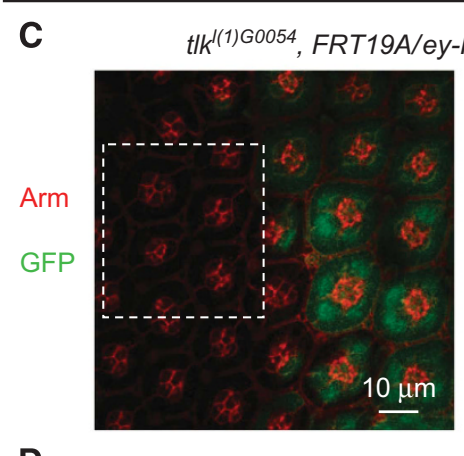

D $_{\text {GMR }>\text { white }}{ }^{R N A i}$

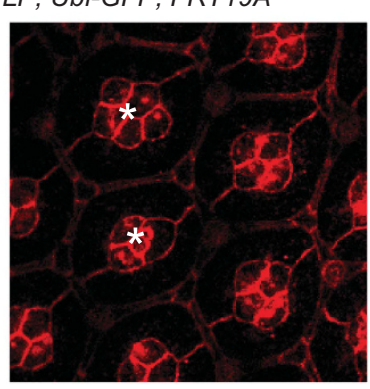

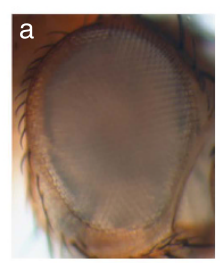

GMR-Gal4/+

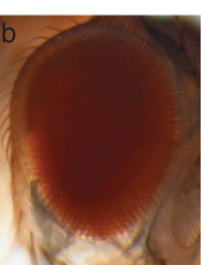

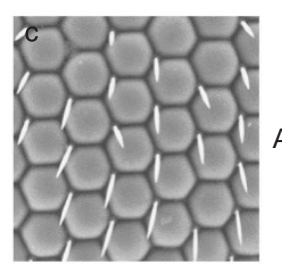

Arm

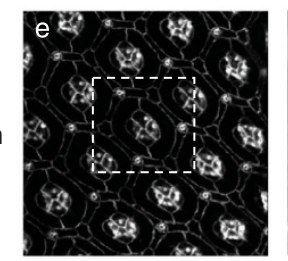

$G M R>t \mid k^{R N A i}$
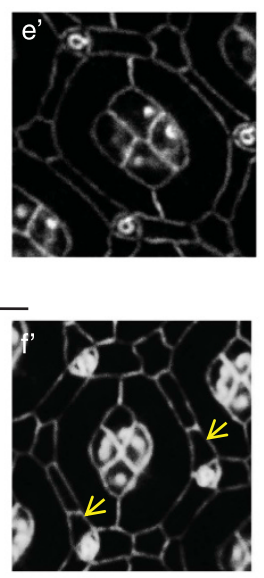

Figure 2 Loss of $t / k$ affects the developmental eye patterning. (A) Immunostaining of the $40 \mathrm{~h}$ APF retina with anti-Armadillo (red) or GFP (green). (a) The wild-type fly, $w^{1118}$. A hexagon is superimposed on the image to indicate the target area, which exemplifies the scoring method. Twenty-one cells lie within a target area of the wild-type fly, including 18 intact cells and six corner (labeled as *) counting as 3 cells. A representative cone cell, bristle cell, primary $\left(1^{\circ}\right)$, secondary $\left(2^{\circ}\right)$ and tertiary $\left(3^{\circ}\right)$ pigment cell are marked on the micrograph. (b) The t/k mutant clones are visualized by the absence of GFP. The wild-type clones and homozygous mutant clones are labeled with $+/+$ and $-/-$, respectively. ( $\mathbf{b}^{\prime}$ ) An enlarged view of the dashed white box in b and the extra IOCs are pointed by white arrows. (B) Quantification of the number of IOCs in A. In all figures, at least 30 hexagonal target areas were scored from three to six flies. (C) Effect of the $t / k$ mutant $\left(t / k^{1(1) G 0054}\right)$ on the development of the cone cells. An enlarged view is shown on the right panel. The wild-type clones always have four cone cells, whereas 20\% mutant clones showed either increased (with five cone cells) or decreased (with three cone cells) number of cone cells, as labeled by the asterisks. (D) Effect of tlk RNAi on the eye development. a and b as a control, the white ${ }^{\text {RNAi }}$ could effectively reduce the pigmentation of the eye compared with its promoter line (GMR-Ga/4). (c and d) The scan electron microscope (S.E.M.) images of the eyes. (e and f) The anti-Armadillo staining. The enlarged views are shown on the right panels (e' and f'), and the extra IOCs are pointed by yellow arrows. (E) Quantification of the number of IOCs in D. (F) Effect of t/k RNAi on the development of perimeter ommatidia. ( $a$ and b) The images of S.E.M. (c and d) Immunostaining of the $40 \mathrm{~h}$ APF retina with anti-Armadillo (red). The arrows point to the extra small perimeter ommatidia. (G) Effect of tlk RNAi on cell proliferation. Immunostaining of the eye disc from the 3rd instar larvae with anti-phospho-pH3 (pH3). The arrow heads point to the morphogenic furrow. The number of pH3-positive cells in the posterior region of the eye disc is counted. The statistic results from five eye discs are shown under each condition. (H) AO staining of retina. Twenty areas in each eye disc were scored, and three to five eye discs were examined. The statistic results from five eye discs are shown under each condition. (I) Genetic interaction between tlk and DIAP1. (J) Quantification of IOCs 
E

\begin{tabular}{lll}
\hline Genotype & $\begin{array}{l}\text { Cells / hexagon } \\
\pm \text { s.e.m. }\end{array}$ & $\begin{array}{l}\triangle \mathrm{IOC} / \text { ommatidium } \\
\pm \text { s.e.m. }\end{array}$ \\
\hline GMR>white & $21 \pm 0$ & $0 \pm 0$ \\
\hline$G M R>t / k^{R N A i}$ & $24.34 \pm 0.96^{* *}$ & $1.11 \pm 0.32$ \\
\hline
\end{tabular}

$\mathbf{F}$
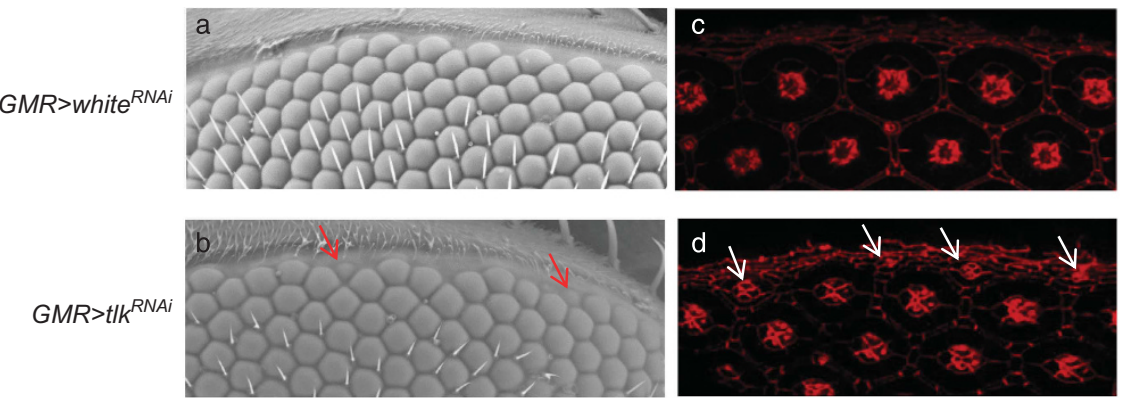

G

GMR>white ${ }^{R N A i}$

$G M R>t I k^{R N A i}$

H GMR>white ${ }^{R N A i}$

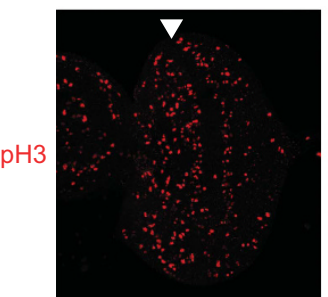

$103 \pm 9.4 /$ disc

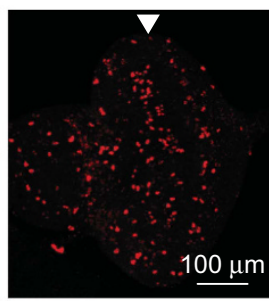

$94 \pm 7.8 /$ disc

I

GMR-DIAP1

Arm

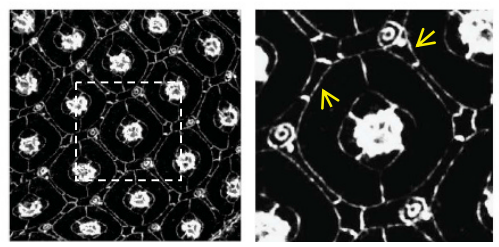

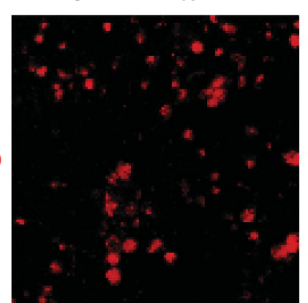

$102 \pm 7.5 / 10^{4} \mu \mathrm{m}^{2}$

$G M R>t I k^{R N A i}$

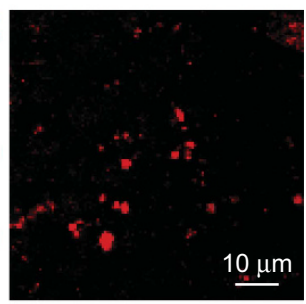

$44 \pm 7.7^{* * *} / 10^{4} \mu \mathrm{m}^{2}$

GMR-Gal4/GMR-DIAP1;UAS-tlk RNAi/+ $^{2}$

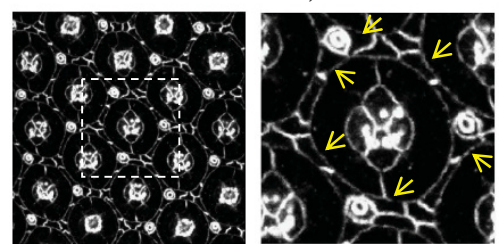

$\mathbf{J}$

\begin{tabular}{lll}
\hline Gepytone & $\begin{array}{l}\text { Cells / hexagon } \\
\pm \text { s.e.m. }\end{array}$ & $\begin{array}{l}\triangle \text { IOC / ommatidium } \\
\pm \text { s.e.m. }\end{array}$ \\
\hline GMR>white & $21 \pm 0$ & $0 \pm 0$ \\
\hline GMR $-D I A P 1$ & $22.25 \pm 0.32^{* *}$ & $0.41 \pm 0.11$ \\
\hline GMR-Gal4/GMR-DIAP1;UAS-tlk & $33.00 \pm 1.69^{* *}$ & $4.00 \pm 0.56$ \\
\hline
\end{tabular}

Figure 2 (Continued)

these possibilities, we examined eye development in the 3rd instar larvae. At this stage, the morphogenetic furrow (MF) marks the forward edge of differentiation, and the cell clusters at different stages of differentiation are aligned posterior to the MF, with the more mature cell clusters located at a further posterior region. ${ }^{29}$ Some cells in the posterior region undergo a secondary division wave. Eventually, clusters of five $R$ cells are formed, followed by recruitment of more $\mathrm{R}$ cells and other cell types at a later developmental stage. ${ }^{31}$ Consistent with the expression of the GMR promoter in the posterior region, ${ }^{28}$ $\mathrm{R}$ cells were present in the eye disc of the $G M R>t l k$ flies ( $R$ cells were labeled with ELAV) (Figure $1 \mathrm{C}$ a and b), suggesting that differentiation was not significantly affected. However, the number of $R$ cells in some clusters was reduced (Figure $1 C b$ ).
Because almost all $R$ cells were missing in the adult stage of GMR $>$ t/k (Figure 1A e and f), the $R$ cells likely started to die at the late larval stage. Therefore, overexpression of $t / k$ promotes cell death instead of affecting differentiation.

TLK regulated $\mathrm{PCD}$ during eye development. When raised at $25^{\circ} \mathrm{C}$, each ommatidium adopts a rigid hexagonal pattern in the wild-type flies at $40 \mathrm{~h}$ APF (after pupa formation), and anti-Armadillo staining can visualize each cell in the developing retina. ${ }^{32}$ To quantify the number of IOCs in the developing retina, a hexagonal target area is defined by connecting the center of six ommatidia surrounding a central ommatidium (Figure $2 \mathrm{~A}$ a). ${ }^{33}$ 
To test whether loss-of-function t/k may promote cell survival in the developing retina, we studied a tlk mutant. As a member of the conserved serine/threonine kinase family, TLK regulates cell cycle progression and chromatin assembly. ${ }^{34} \mathrm{~A}$ P-element-mediated loss-of-function mutant, $t / k^{\prime(1) G 0054}$, has been well characterized, and it induces DNA fragmentation, cell death and pupal lethality. ${ }^{34}$ To determine the effect of this mutant on the Drosophila eye, we performed mosaic analysis. In the pupal retina of the mosaics, the wildtype clones were labeled with two copies of GFP, whereas the homozygous mutant $t / k\left(t / k^{\prime(1) G 0054} / t / k^{\prime(1) G 0054}\right)$ cells displayed no GFP labeling (Figure 2A b). Strikingly, the tlk homozygous mutant clones showed a significant increase in the number of IOCs (Figures 2A b' and 2B). In addition, $\sim 20 \%$ of the ommatidia developed more or fewer cone cells (3 or 5 compared with 4 in the wild-type) in the tlk homozygous mutant clones (Figure 2C, the asterisks labeled ommatidia). This result indicated that the cone cell division may be affected in the tlk homozygous mutant. Because cone cells can affect the recruitment and survival of IOCs, ${ }^{2,8}$ the defects in the cone cells may complicate the interpretation of TLK function in the IOCs. To determine the function of TLK at a later developmental stage, we examined the tlk RNAi lines driven by GMR-Gal4. A genetically matched line, white ${ }^{R N A i}$, was used as the control. The white ${ }^{R N A i}$ line was functional because it can efficiently reduce the pigmentation of GMR-Gal4 (Figure 2D a and b). In the GMR $>t / k^{R N A i}$ adult eyes, the shape of the ommatidia were irregular, indicating defects in the patterning of the eyes (Figure 2D $c$ and d). To quantify the defect, we examined the pupal retina at $40 \mathrm{~h}$ APF. The result showed that extra IOCs (Figures 2D e, f and 2E) and perimeter ommatidia (Figure 2F) were present. Therefore, extra IOCs survived with the loss of $t / k$.

To determine whether the extra IOCs resulted from reduced cell death or increased proliferation, the eye discs were stained with anti-phospho-histone $\mathrm{H} 3(\mathrm{pH})$, a marker of cell proliferation. ${ }^{35}$ The result showed that the $\mathrm{pH} 3$ staining pattern was unaltered in the 3rd instar larval eye disc of the $G M R>t / k^{R N A i}$ lines (Figure $2 \mathrm{G}$ ), indicating that the extra number of IOCs and perimeter ommatidia were not due to increased cell proliferation. To further test whether PCD was reduced in the GMR $>t / k^{R N A i}$ lines, we stained the pupal retina (at $35 \mathrm{~h}$ APF) with acridine orange (AO), a marker of cell death. ${ }^{36}$ The result showed reduced $A O$ staining in the retina of the $G M R>t I k^{R N A i}$ flies, compared with the control retina (Figure 2H). Therefore, TLK mediated PCD during eye patterning.

The canonical caspase pathway is the main executioner of PCD in eye patterning. ${ }^{2}$ To test the genetic interaction between TLK and the caspase pathway, we examined the effect of overexpression of DIAP1 on IOCs. DIAP1 blocks the executioner caspases, including DrICE and Dcp $-1 .{ }^{9}$ The result showed that the tested fly (GMR>DIAP1/tIk $\left.{ }^{R N A}\right)$ eyes exhibited even more IOCs than either $G M R>t k^{R N A i}$ or GMR-DIAP1 alone (Figure $2 \mathrm{l}$ and J), suggesting that TLK mediated a new type of PCD that is independent of the apoptotic pathway.

Characterization of TLK-induced cell death. To study TLK-induced cell death, we stained the eye discs of the 3rd instar larvae with AO (Figure 3A a-c). GMR-hid was used as a positive control (Figure $3 \mathrm{~A} \mathrm{c}$ ). Interestingly, the $\mathrm{AO}$ staining intensity was even stronger in the $G M R>t / k$ flies than the $G M R>$ hid flies, suggesting that ectopic expression of $t / k$ can strongly induce cell death. Further, we examined DNA fragmentation by TdT-mediated dUTP nick end labeling (TUNEL) (Figure $3 \mathrm{~A} d-f)$. The results demonstrated intensely labeled TUNEL signals (Figure 3A e). Because TUNEL can also label necrotic cells, in which the membrane integrity is disrupted, ${ }^{37}$ we tested whether the membrane was intact by $\mathrm{PI}$ (propidium iodide) staining (Figure $3 \mathrm{~B}$ a-c). The results showed that the PI signal was negative in the GMR $>t / k$ flies (Figure $3 \mathrm{~B} \mathrm{~b}$ ). As a positive control, the necrosis model of sev $>$ GlutR $1^{L C} 37$ was PI positive (Figure $3 B \mathrm{c}$ ). These results suggested that overexpression of TLK was sufficient to induce cell death.

Next, we asked whether the apoptotic pathway was involved in the cell death of the GMR $>$ tlkflies. The results showed that inhibition of the caspase pathway by overexpression of $p 35$, $D I A P 1$ and DIAP2 had no effect on the eye defect in the $G M R>t / k$ flies (Figure 4A). As positive controls, these lines suppressed apoptosis in the GMR-hid fly (Supplementary Figure S2). Moreover, we examined an in vivo sensor of caspase activation, Apoliner, which comprises two fluorophores: enhanced green fluorescent protein (eGFP) and monomeric red fluorescent protein (mRFP). ${ }^{38}$ These fluorescent proteins are linked by a peptide sequence containing a caspase-sensitive cleavage site. Upon caspase activation, the cleavage of Apoliner allows the eGFP to translocate into the nucleus, whereas the mRFP remains on the cell membrane. ${ }^{38}$ As a positive control, the mRFP was presented on the cell membrane in the posterior region of the eye disc in the GMR-hid flies (Figure 4B b). In contrast, the eye disc of $G M R>t l k$ flies showed no enhanced presence of the mRFP on the cell membrane (Figure 4B c). In addition, we performed immunostaining using the cleaved Dcp-1 antibody (Figure $4 \mathrm{~B} d-f$ ). The results showed that there was no signification activation of the caspase activity in the GMR $>t / k$ flies compared with the control flies (Figure 4B f). Together, these results suggest that the TLK-induced cell death was independent of caspase function.

JNK is known as a mediator of caspase-independent cell death in Drosophila. ${ }^{16,18}$ We observed that inhibition of the JNK pathway by overexpression of a dominant-negative Bsk $\left(B s k^{D N}\right)$ did not suppress the eye defect of $G M R>t l k$ flies (Figure 4C). Consistently, the JNK pathway was not activated in the eye disc of GMR > tlkflies as determined by puc-lacZ, an in vivo reporter of JNK activation ${ }^{39}$ (Figure $4 \mathrm{D} a-b$ ). In contrast, the positive control of GMR $>$ eiger ${ }^{17}$ showed robust induction of the JNK signal (Figure 4D c). AIF is a mediator of caspase-independent cell death in Drosophila. ${ }^{18}$ We found that loss-of-function of AIF had no effect on the cell death in GMR>tlk flies; a similar effect was observed with the p53 mutant or overexpression of ROS chelating enzymes (catalase and GTPx-1) (Figure 4E).

TLK has an important role in chromatin assembly, including chromosome segregation, replication, transcription and DNA repair. ${ }^{40}$ In Drosophila, TLK phosphorylates ASF1 and causes nuclear division arrest and pupal lethality. ${ }^{34}$ Our result showed that asf $1^{R N A i}$ had no effect on the TLK-induced cell death 
A

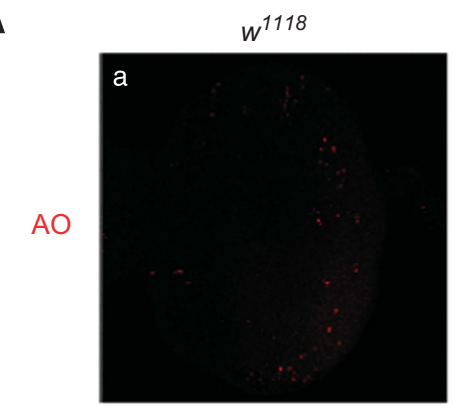

$7.5 \pm 3.5 /$ disc
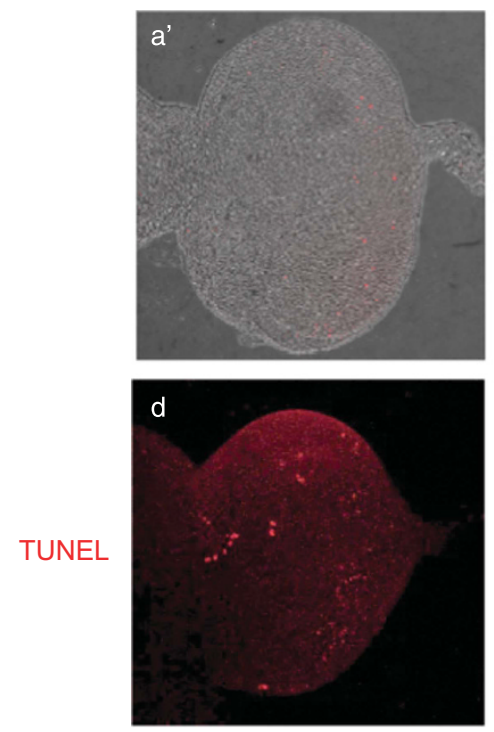

$9.2 \pm 6.0 /$ disc

B
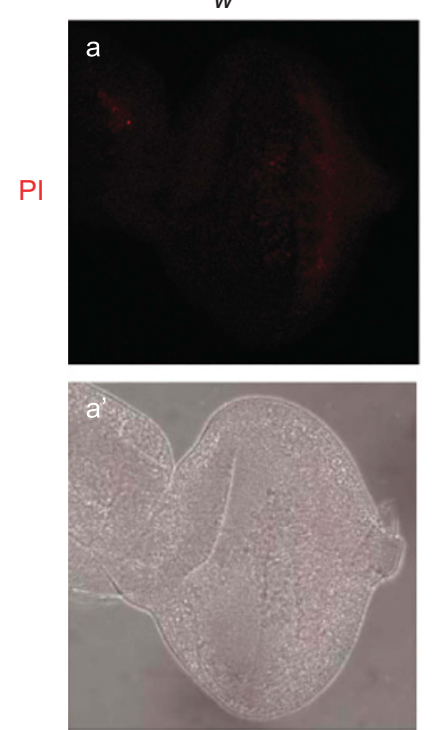

$G M R>t l k$

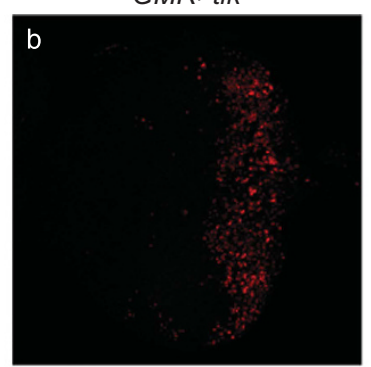

$252.3 \pm 33.8 /$ disc
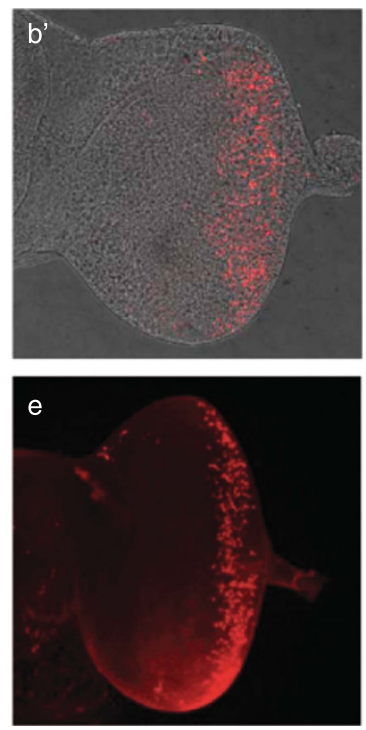

$240.4 \pm 20.5 /$ disc

GMR>tlk
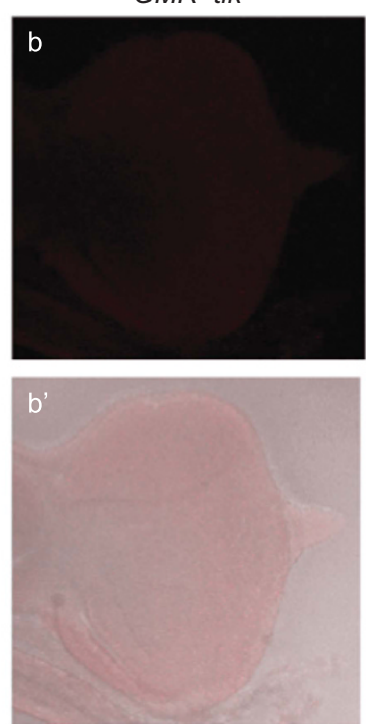

GMR-hid

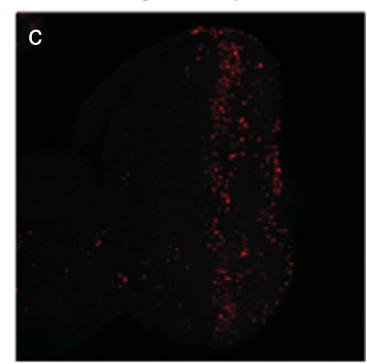

$140.3 \pm 8.1 /$ disc
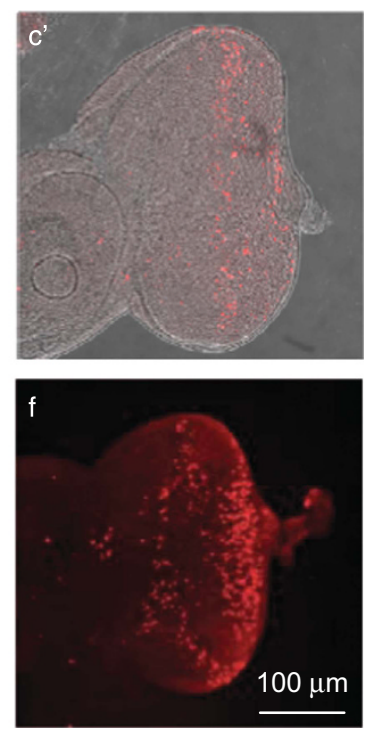

$152.6 \pm 12.8 /$ disc
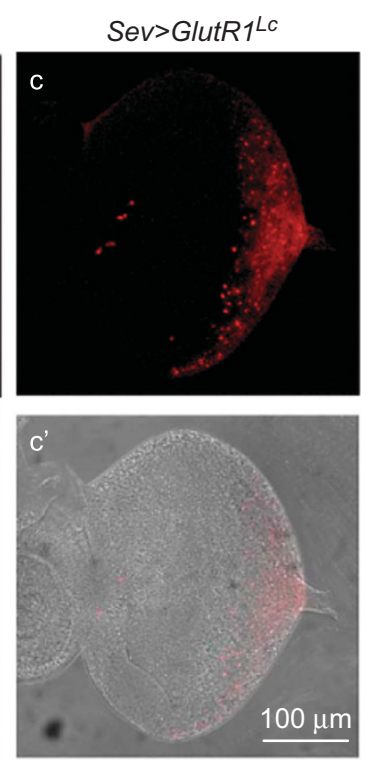

Figure 3 Cell death induced by ectopic expression of $t / k$ in Drosophila eye. (A) AO and TUNEL staining of the eye disc from the 3rd instar larvae. GMR-hid is shown as a positive control. $(a-c)$ The AO staining. $\left(a^{\prime}-c^{\prime}\right)$ The merged image of bright field and AO staining. (d-f) TUNEL staining. The integrative density (Intden) of the posterior region in each eye disc was obtained by ImageJ. Five eye discs were examined for each genotype. (B) PI staining of the eye disc. (a-c) PI staining. (a'-c') Merged image of bright field with $\mathrm{PI}$ staining. The sev $>$ GlutR $1^{L C}$ is shown as a positive control 
A

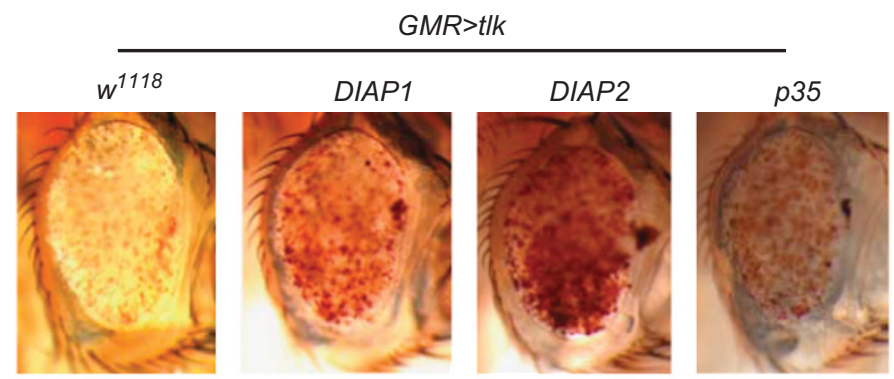

B

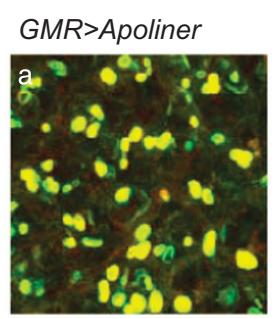

$w^{1118}$

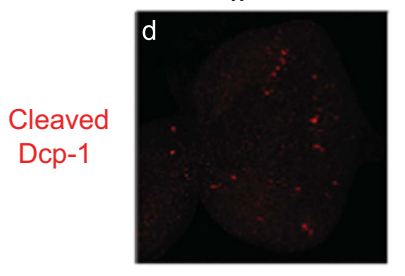

$22 \pm 3.4 /$ disc

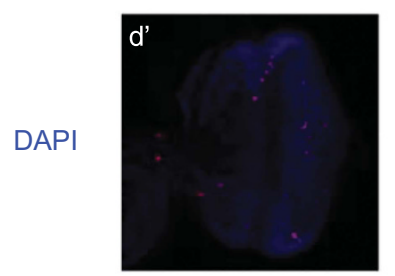

C
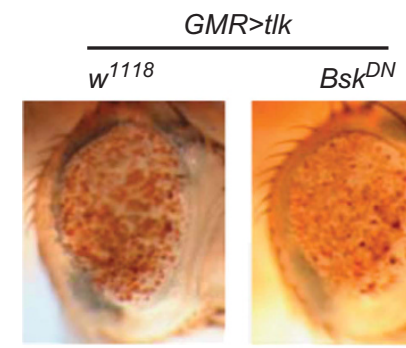

E

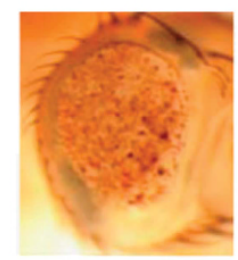

GMR-Gal4, GMRhid/UAS-Apoliner

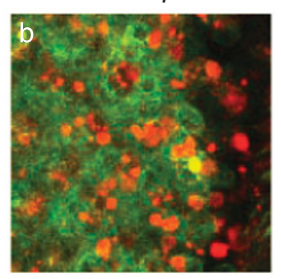

GMR-hid

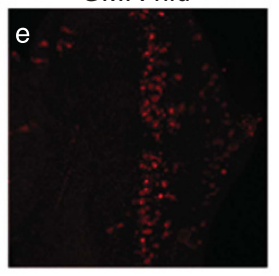

$197 \pm 20.2 /$ disc

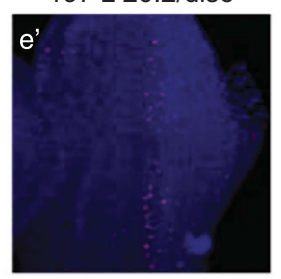

D
GMR-Gal4/UAS-

Apoliner; UAS-tlk/+

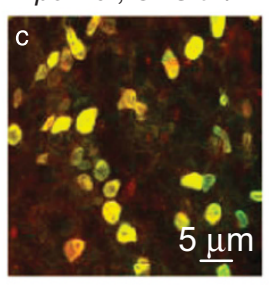

GMR $>$ tlk

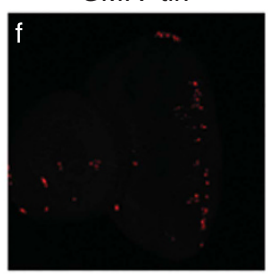

$28 \pm 10.5 /$ disc

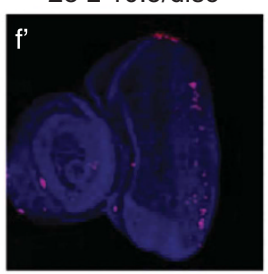

puc-lacZ

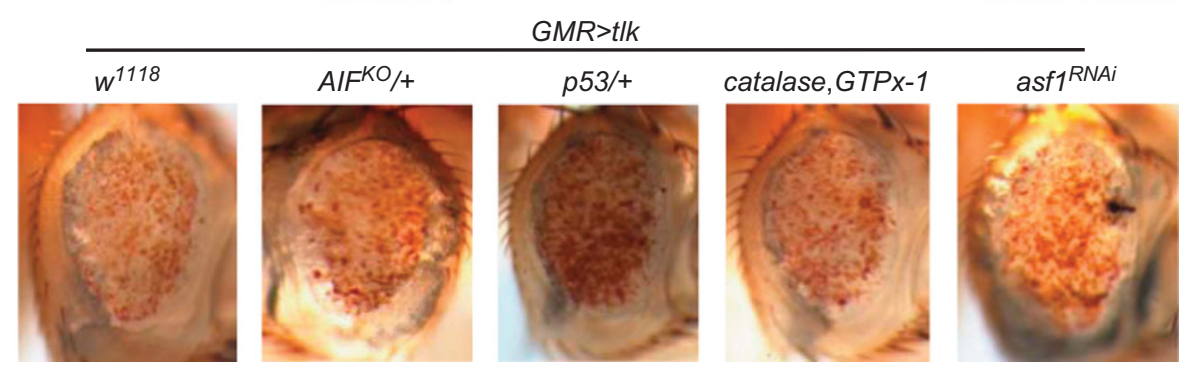

Figure 4 Characterization of the TLK-induced cell death. (A) Effect of inhibition of caspase pathway on the GMR $>$ tlk. (B) Caspase activity in the larval eye disc. (a, b and c) Alteration of apoliner fluorescence. (d, e and f) Immunostaining with anti-cleaved Dcp-1. The number of positive dots in the posterior region of the eye disc is counted. GMR-hid is shown as a positive control. The statistic results from five eye discs are shown under each condition. $d^{\prime}, e^{\prime}$ and $f^{\prime}$ are merged images of DAPI and cleaved Dcp-1 staining. (C) Effect of inhibition JNK signaling pathway on GMR > tlk. (D) JNK activity in the larval eye disc. Puc-lacZ is an in vivo reporter of JNK activation that can be detected by the X-gal staining. The GMR > eiger is shown as a positive control. (E) Effect of several genes related to cell death or chromatin assembly on GMR $>$ tlk eye defects 
(Figure 4E), suggesting that the function of TLK on cell death was likely different from its role in chromatin organization. Together, these results suggested that TLK may induce a new cell death pathway.

ATG2 functioned downstream of the TLK-mediated PCD. To understand the molecular mechanism behind TLKinduced cell death, we screened $\sim 1000$ TRiP RNAi lines using the GMR $>$ tlk flies. These RNAi lines are inserted into a defined genomic locus, thus eliminating the variation of positional effects compared with random P-element insertions. $^{30}$ We identified nine suppressor and enhancer lines (Table 1). Most of the suppressors are components of the mediator family, which affected the transcription of $t / k$. In contrast, atg2 RNAi did not affect the transcription of $t / k$ and was further studied. The $\operatorname{atg} 2^{R N A i}$ showed a strong rescue effect on the GMR > tlk flies (Figure 5A a and b). Moreover, $\operatorname{atg} 2^{R N A i}$ reduced $A O$ and TUNEL staining in the tlk-overexpressing eye discs (Figure 5B), suggesting that cell death was suppressed. Interestingly, the number of IOCs was also increased at $40 \mathrm{~h}$ APF in the retina of the $G M R>\operatorname{atg} 2^{R N A i}$ flies (Figure $5 \mathrm{C}$ a). The efficiency of the $\operatorname{atg} 2^{R N A i}$ was confirmed (Supplementary Figure S3). Using the CRISPRassociated single-guide RNA system (Cas9/sgRNA), ${ }^{41}$ we generated a large deletion (2164 bp) in the exon region of the atg2 gene. The homozygous deletion was pupal lethal. Therefore, we generated mosaics in the fly eyes. Similar to the RNAi effect, the clones of the homozygous deletion displayed a significant increase in the number of IOCs (Figure $5 \mathrm{C}$ b). In addition, knocking down both tlk and atg2 by RNAi resulted in a similar number of IOCs as the $G M R>t l k^{R N A i}$ alone (Figure $5 \mathrm{C} \mathrm{c}$ ), suggesting that ATG2 acts in the same pathway with TLK. Moreover, atg $2^{R N A i}$ had a synergistic effect with GMR-DIAP1, similar to $t / k^{R N A i}$ (Figure $5 \mathrm{C} d$ ). The statistical results are presented (Figure 5D). Because ATG2 is known to be involved in autophagy, ${ }^{19}$ we asked whether autophagy was activated. There was no LysoTracker staining in the larval eye disc of the $G M R>t l k$ flies (Supplementary Figure S4 a and b). As a positive control, the eye disc of sev $>$ GlutR $1^{L C}$ showed intense LysoTracker staining (Supplementary Figure S4 c), as reported previously. ${ }^{37}$ We also tested several autophagyrelated genes by $\mathrm{RNAi}$, including atg1, atg4, atg8a, atg9 or atg16. The result showed that none of the genes rescued the eye defect of the GMR > tlk flies (Supplementary Figure S5). Together, these results suggested that ATG2 might function downstream of TLK.
To explore the mechanism of TLK-induced cell death, we stained the eye disc of $G M R>$ tlk-flag with a nuclear membrane marker (NPC), anti-Flag and DAPI. In the GMR $>$ tlk-flag cells, TLK-Flag was stained in the nucleus (Supplementary Figure S6a and b), as reported. ${ }^{34}$ Meanwhile, the nuclear membranes were damaged or had disappeared in some of the tlk-expressing cells (Supplementary Figure S6b). Because TLK is a nuclear protein, it was likely that the cell death caused dysfunction of the nucleus.

TLK mediated mild calcium overload-induced cell death. Our data suggested that TLK may induce a new type of PCD in Drosophila eye development. Does TLK also function in pathological conditions? Previously, we were interested in calcium overload-induced cell death and generated a transgenic Drosophila line to express GluR1 ${ }^{L c}$ (rat glutamate receptor 1 lurcher mutant), ${ }^{37}$ a constitutively open cation channel in vitro. ${ }^{42}$ Another transgenic line that we generated is $h s-G l u R 1^{L c}$, in which GluR1 $1^{L C}$ is driven directly by a heat shock (hs) promoter. These flies have defects in the eyes when raised at $25^{\circ} \mathrm{C}$ (Figure $6 \mathrm{~A}$ a and $\mathrm{d}$ ). To further assess cell death, the fly eyes were sectioned (Figure 6A b, c, e and f). We observed that some ommatidia disappeared and were accompanied by the presence of large vacuoles throughout the eyes in the 1-day-old $h s-G l u R 1^{L c}$ flies (Figure $6 \mathrm{~A} \mathrm{e}$ ), and no cellular structure was observed in the 10-day-old $h s-G l u R 1^{L c}$ flies (Figure 6A f). These results suggested that mild calcium overload induced cell death in the Drosophila eyes. To quantify calcium overload, we measured the intracellular calcium levels in the 3rd instar larval eye disc using Fura-2. Indeed, the calcium levels were increased by $\sim 20 \%$ in the $h s$-GluR $1^{L C}$ flies compared with the control flies (Figure 6B).

Next, we investigated the cell death pathways involved in the $h s-G l u R 1^{L c}$ flies. The results showed that $t k^{R N A i}$ strongly rescued the eye defect of the $h s-G l u R 1^{L c}$ flies (Figure $6 \mathrm{C} \mathrm{b}$ and c). For the other pathways, suppression of the caspasemediated pathway had a partial rescue effect, whereas the other pathways, including AIF, JNK and autophagy, showed no effect (Supplementary Figure S7). In addition, $\operatorname{atg} 2^{R N A i}$ showed a partial rescue of the $h s-G l u R 1^{L C}$ eyes (Figure $6 \mathrm{C} d$ and $h$ ). Although TLK may regulate transcription, ${ }^{43}$ the transcription of $G l u R 1^{L c}$ was not affected by the $t / k^{R N A i}$ lines (Figure 6D). Therefore, the TLK-mediated cell death was active in the mild calcium overload condition in the fly eyes.

Table 1 Genetic modifiers of cell death in $G M R>t / k$

\begin{tabular}{llll}
\hline CG number & Gene symbol & Gene function & Effect \\
\hline CG13867 & MED8 & RNA polymerase II transcription cofactor activity & Rescue \\
CG9936 & Skd/MED13 & RNA polymerase II transcription cofactor activity & Rescue \\
CG5546 & RED19 & RNA polymerase II transcription cofactor activity & Rescue \\
CG17397 & RED21 & RNA polymerase II transcription cofactor activity & Rescue \\
CG5121 & MED28 & RNA polymerase II transcription cofactor activity & Rescue \\
CG17183 & MED30 & RNA polymerase II transcription cofactor activity & Rescue \\
CG1241 & Atg2 & Autophagy & Enhance \\
CG43665 & elF-2 & GTPase activity & Enhance \\
CG6779 & RpS3 & Structural constituent of ribosome &
\end{tabular}


A

GMR>tlk
\[ \operatorname{attP2} \]

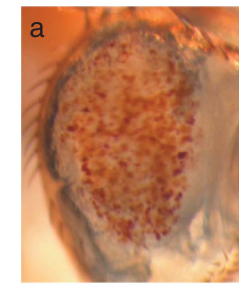

B

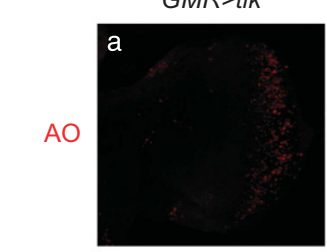

$230.4 \pm 36.9 /$ disc

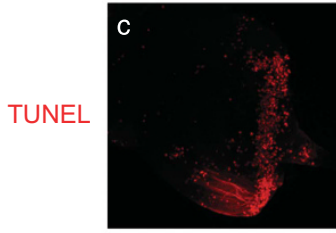

$250.0 \pm 30.2 /$ disc

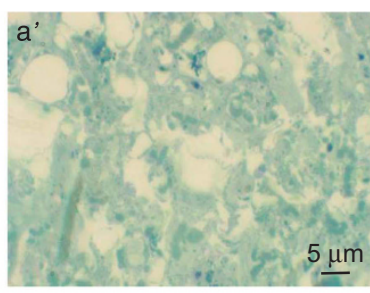

GMR-Gal4/+; UAS-t/k/UAS-atg $2^{R N A}$

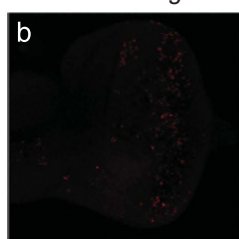

$69.2 \pm 13.0^{* * *} / \mathrm{disc}$

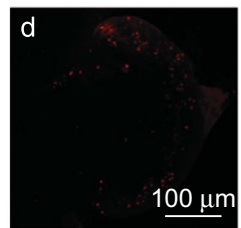

$50.1 \pm 14 \cdot 1^{* * *} /$ disc

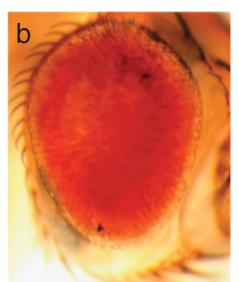

C

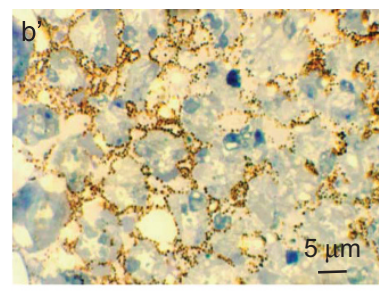

$G M R>\operatorname{atg} 2^{R N A i}$
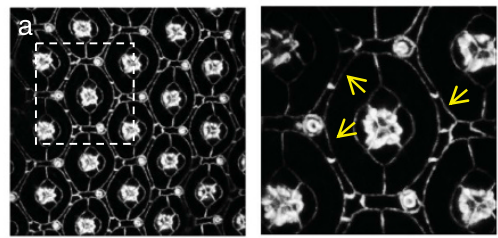

ey-FLP/+;; $\Delta$ atg2, FRT80B/ Ubi-GFP, FRT80B

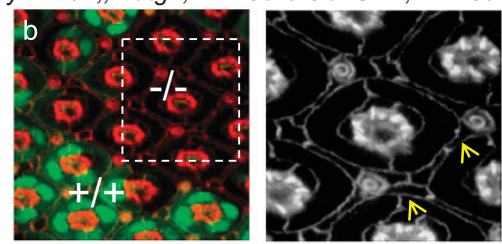

GMR-Gal4/+; UAS-tlk ${ }^{R N A i} / U A S-a t g 2^{R N A i}$

\section{D}

\begin{tabular}{|c|c|c|}
\hline Genotype & $\begin{array}{l}\text { Cells / hexagon } \\
\pm \text { s.e.m. }\end{array}$ & $\begin{array}{l}\triangle \triangle \mathrm{IOC} / \\
\text { ommatidium } \\
\pm \text { s.e.m. }\end{array}$ \\
\hline$G M R>\operatorname{atg} 2^{R N A i}$ & $22.46 \pm 0.82^{\mathrm{a}}$ & $0.48 \pm 0.27$ \\
\hline $\begin{array}{l}\text { ey-FLP/+;; } \triangle a t g 2, \\
\text { FRT80B/ Ubi-GFP, } \\
\text { FRT80B }\end{array}$ & $22.01 \pm 0.67^{\mathrm{a}}$ & $0.34 \pm 0.22$ \\
\hline $\begin{array}{l}\text { GMR-Gal4/+; UAS- } \\
\text { tlk }^{\text {RAI }} / \cup A S-\operatorname{atg2} 2^{\text {RAAi }}\end{array}$ & $24.50 \pm 0.75^{a, b}$ & $1.17 \pm 0.25$ \\
\hline $\begin{array}{l}\text { GMR-Gal4/GMR- } \\
\text { DIAP1;UAS-atg2 }{ }^{\text {RAAi } /+}\end{array}$ & $28.77 \pm 0.43^{\mathrm{a}, \mathrm{b}}$ & $2.59 \pm 0.14$ \\
\hline
\end{tabular}

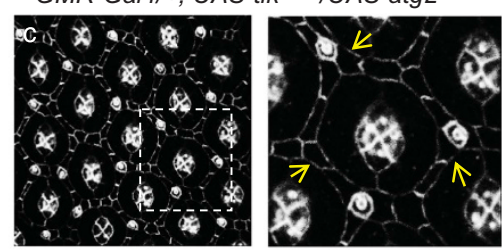

GMR-Gal4/GMR-DIAP1;UAS-atg2 $2^{\text {RNAi//+ }}$
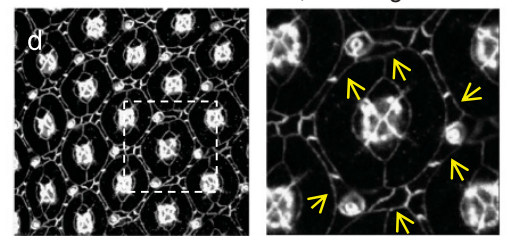

Figure 5 Genetic interaction between atg2 and t/k. (A) Effect of atg $2^{R N A i}$ on GMR $>$ tlk. (a and b) The light image. ( $a^{\prime}$ and b') The toluidine blue-stained semi-thin sections of the eyes. (B) AO and TUNEL staining. The Intden of the posterior region was obtained by ImageJ. Five eye discs were examined for each genotype. (C) Effect of loss of atg2 on the IOC number. The genotypes are indicated on top of each micrograph. Enlarged views of the dashed white box are shown in the right panel, and the yellow arrows point to the extra IOCs. The atg2 deletion mutant clones are visualized by the absence of GFP. The wild-type clones and homozygous mutant clones are labeled with $+/+$ and $-/-$, respectively. (D) Quantification of the number of IOCs in C. Compared with the control fly (GMR> white ${ }^{R N A}$ ), all these lines showed statistical difference, and is denoted as 'a'. Compared with $G M R>\operatorname{atg} 2^{R N A i}$, the lines with statistical difference is denoted as 'b'

\section{Discussion}

Previous studies have demonstrated that TLK has a crucial role in chromatin assembly, including transcription, replication, DNA damage repair and chromosome segregation. ${ }^{40}$ As a kinase, TLK functions by phosphorylating its target proteins, such as the chromatin assembly factor ASF1 and Rad9. ${ }^{40}$ In the Drosophila loss-of-function t/k mutant, nuclear divisions are arrested at interphase, and cells eventually undergo apoptosis. ${ }^{34}$ Therefore, loss-of-function tlk should reduce cell numbers. However, we observed an increased number of IOCs in the t/k homozygous mutant clones and in the
$G M R>t / k^{R N A i}$ fly eyes. This suggests that $t / k$ function in PCD must be unrelated to its role in cell division. Consistently, the function of TLK in chromatin assembly requires ASF $1{ }^{34}$ however, afs $1^{R N A i}$ had no effect on TLK-induced cell death. Therefore, the role of TLK in cell death may be less likely through its function in chromatin assembly.

Our data show that TLK participates in the process of PCD during Drosophila eye development. In support of this, both the t/k mutant and two independent RNAi lines could reduce cell death in IOCs without affecting cell proliferation. In addition, $t / k$ overexpression is sufficient to induce cell death. 
A

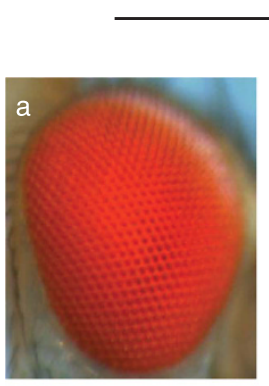

B

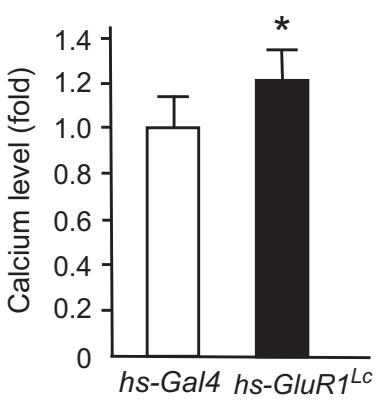

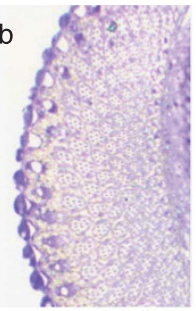

10 day

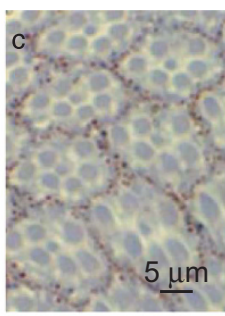

$\mu \mathrm{m}$
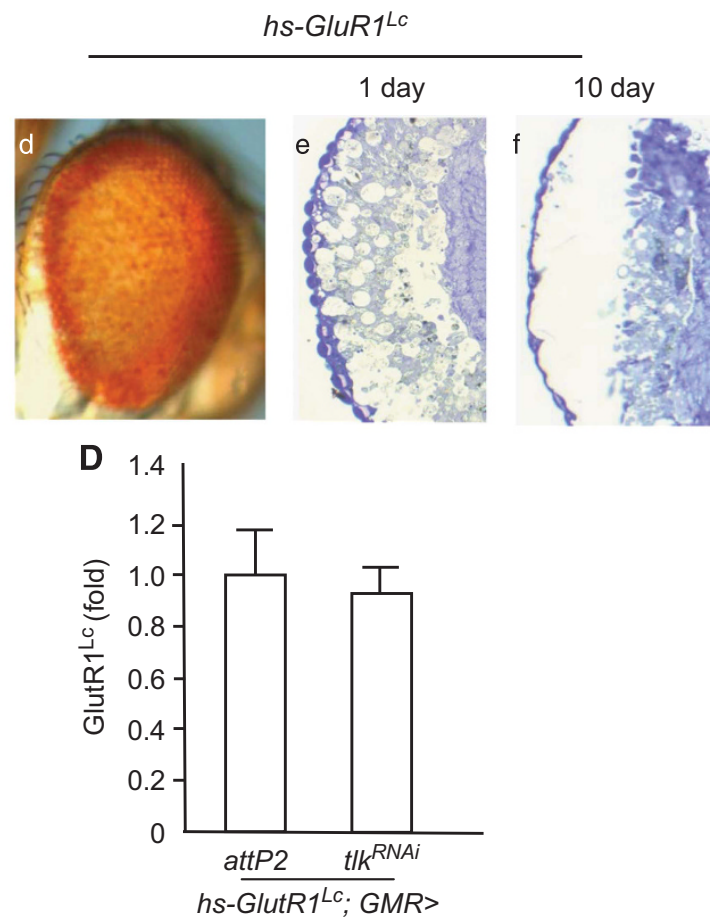

C

hs-GlutR1 ${ }^{L c}$; GMR>
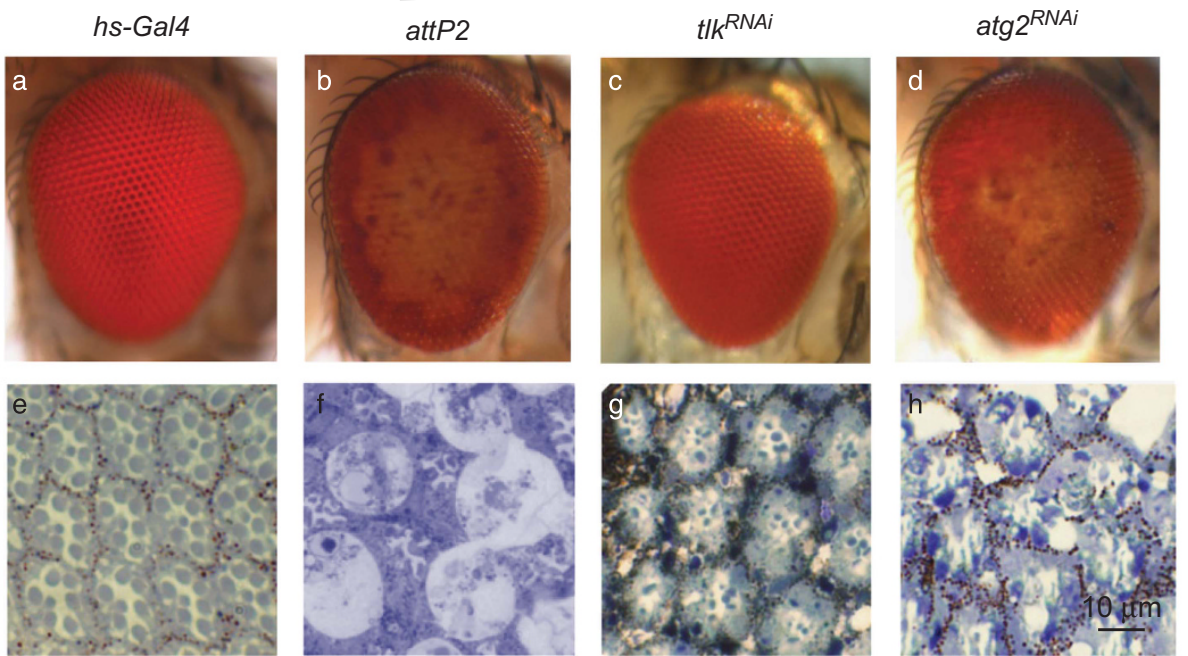

Figure 6 The $h s-G l u R 1^{L C}$ model and the effect of $t / k^{R N A i}$. (A) The pigmentation defect in the eye of $h s$-GluR1 ${ }^{L c}$. Light images and toluidine blue-stained semi-thin sections are shown. (B) Intracellular calcium level in the larval eye disc measured by Fura-2. Trial $n=3$. Six larvae were examined for each trial. The open bar indicates the control, whereas the black bar indicates statistically significant difference. ${ }^{*}$ represents $t$-test, $P<0.05$. (C) Effect of $t k^{R N A i}$ and atg ${ }^{R N A i}$ on the $h s$-GluR1 ${ }^{L C}$ eye defect. The upper panels are the

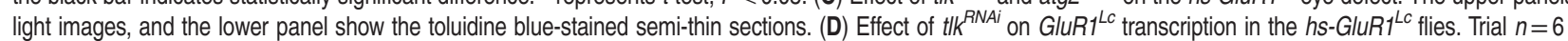

In theory, $t / k^{R N A i}$ might promote a reduction of PCD by enhancing survival signals, such as EGF and Ras, or suppressing death signals, such as Notch. ${ }^{2,8}$ Because these pathways partially converge onto the apoptotic pathway to regulate eye patterning, ${ }^{44-46}$ the independence of $t / k^{R N A i}$ with respect to DIAP1 function suggests that this possibility is less likely. Moreover, we find that TLK-induced cell death cannot be rescued by most of the known cell death pathways, including caspase, autophagy, AIF, JNK, ROS and $\mathrm{p53}$. Therefore, we propose that TLK regulates a new type of PCD.
It is not clear how TLK causes cell death. Interestingly, the nuclear membrane seems severely disrupted in TLKoverexpressing cells. Because TLK is located in the nucleus, it may phosphorylate nuclear substrates and promote the disruption of the nuclear membrane. On the other hand, ATG2 is known to function in the formation of the autophagosome membrane, ${ }^{47}$ but may also function in the maintenance of the nuclear membrane. This hypothesis requires further investigation.

In addition to regulating developmental PCD, TLK may also be involved in disease. Our data suggest that the cell death 
observed in the $h s-G l u R 1^{L c}$ fly eye is partially regulated by the apoptotic pathway. However, this calcium-induced cell death was strongly suppressed by the $t / k^{R N A i}$ lines, indicating that TLK may function in calcium overload-induced cell death. Mild calcium overload has been implicated in human diseases, such as Alzheimer's disease and glaucoma. ${ }^{25,48}$ The potential function of TLK in these diseases requires further study. In addition, how TLK may be activated in vivo remains unclear.

In conclusion, we have identified a potential novel cell death pathway that functions in both eye development and calcium cytotoxicity. Strikingly, TLK is both necessary and sufficient for this variant of cell death.

\section{Materials and Methods \\ Drosophila stocks and maintenance. Drosophila stocks were maintained at $25^{\circ} \mathrm{C}$ on standard fly food. The following fly strains were kindly provided by different labs: $p u c^{E 69}$-lacZ (Dr. Tian Xu); AIF ${ }^{K O}$ (Dr. Josef Penninger); UAS-IAP2 (Dr. Pascal Meier); UAS-catalase and UAS-GTPx-I (Dr. Utpal Banerjee); UAS-eiger (Dr. Lei Xue); GMR-hid,GMR-Gal4 (Dr. Andreas Bergmann); p53/Tb (Dr. John Abrams) TRiP RNAi stocks were obtained from the Tsinghua Drosophila stock center: t/k ${ }^{\text {RNAi }}$ (RNAi-1: THU0178, RNAi-2: THU1326); Atg2 $2^{\text {RNAi }}$ (THU3698). All the other lines were obtained from the Bloomington Stock Center, including $t k^{(1) G 0054}$ (BL11593); UAS-Apoliner (BL32122). For the RNAi lines, $y^{1} v^{1} ;$ P $\{$ CaryP $\} a t t P 2$ (BL36303) and white ${ }^{R N A i}$ (BL33623) were used as the genetic background matched controls.}

Generation of a transgenic fly line. The GluR $1^{L C}$ cDNA was digested from pCAGGS-mGluR1 ${ }^{\mathrm{Lc}}$ construct (a gift from Dr. Michisuke Yuzaki) and subcloned into the $p C a S p e R$ - $h s / a c t$ vector. The $h s-G / u R 1^{L c}$ transgenic fly was generated in a $w^{1118}$ background using the P-element-mediated transformation.

Generation of atg2 deletion. The atg2 deletion mutant was generated using the Cas9/sgRNA system. To generate gene deletion, two sg-RNAs were designed to target the atg2 locus. The sg-RNAs sequences that flank a $2164 \mathrm{bp}$ exon region of the atg2 gene are the following: g1, 5'-GGATGGCTACCACATTCGACCGG-3', g2, 5'-GGATGTCTCCGATGACCGAGGG-3'. The deletion of 2164 bp was confirmed by PCR sequencing.

Histology of adult eyes. The adult fly heads were removed from the body and fixed in the fixative containing $4 \%$ paraformaldehyde and $2.5 \%$ glutaraldehyde in $0.1 \mathrm{M} \mathrm{PB}$, post-fixed in $1 \%$ osmium tetroxide, dehydrated in an ethanol series followed by exchange with propylene oxide. Then, the heads were infiltrated in a mixture of propylene oxide and Spurr's medium, and finally imbedded in Spurr's medium as previously described. ${ }^{49}$ The eyes were semi-thin sectioned to $1.5 \mu \mathrm{m}$ using an ultramicrotome (UC7; Leica, Tokyo, Japan) and stained with toluidine blue.

Histology of larval tissue. AO, immunostaining, $\mathrm{PI}$, lacZ and lysotracker staining were performed as described. ${ }^{36,37,50,51}$ For TUNEL staining, the procedures were followed by the manual of the manufacture (In Situ Cell Death TMR, Product No. 12156792910, Roche, Indianapolis, IN, USA), except an additional treatment of protease $\mathrm{K}$ before the enzymatic reaction. The quantification analysis was performed using ImageJ.

Antibodies used include anti-ELAV (9F8A9, DSHB, lowa, IA, USA; 1:1000), anti-Armadillo (N27A1, DSHB,1:2), anti-phospho-Histone-H3 (06-570, Millipore, Billerica, MA, USA; 1 : 1000), and anti-GFP (A-11122, Thermo Fisher, Rockford, IL, USA; 1 : 1000), anti-cleaved Drosophila Dcp-1 (Asp216) (\#9578, CST, Danvers, MA, USA; 1 : 100), anti-Flag (\#2368, CST; 1 : 1000), anti-Nuclear Protein Complex (NPC) (ab24609, Abcam, Cambridge, MA, USA; $1: 1000$ ).

Fura-2 measurement. The eye discs of the 3rd instar larvae were dissected and incubated with $5 \mu \mathrm{M}$ Fura-2 AM (F-1221, Invitrogen Molecular Probe, Eugene, OR, USA) in Schneider's medium at $25^{\circ} \mathrm{C}$ for $30 \mathrm{~min}$ in the dark. With the excitation wavelengths at 340 and $380 \mathrm{~nm}$, we recorded two emission intensity values at $510 \mathrm{~nm}$, respectively. The ratio of emission intensity at $510 \mathrm{~nm}$ excited by 340 or $380 \mathrm{~nm}$ was calculated as the relative calcium concentration.
qRT-PCR method. Total RNA was extracted by Trizol reagent (Invitrogen, Eugene, OR, USA) followed by DNase I treatment based on the manufacturer's standard protocol, then the purity and integrity of total RNA was determined by $1 \%$ agarose gel electrophoresis. The concentration of total RNA was measured by Nanodrop. Two microgram mRNA was reverse transcribed into cDNA library by oligo-dT primer using RevertAid First Strand cDNA Synthesis Kit (Thermo scientific) based on the manufacturer's standard protocol. The final volume of q-PCR reaction was $25 \mu \mathrm{l}$ using Platinum SYBRGreen qPCR SuperMix-UDG Kit (Invitrogen) containing $1 \mu$ l diluted CDNA sample $(1: 3)$. The q-PCR was performed in triplicate using 7500 real time PCR system (Thermo Fisher). The quantification of target gene was conducted by $\triangle \triangle \mathrm{Ct}$ method. ${ }^{52}$ Primers used are as following:

GAPDH F: 5'-CGCAGCGCCATTCTCCTA-3';

R: 5'-GACTGCCGCTTTTTCCTTTTC-3'

t/k F: 5'-GGGCGGGAACCTACTGGTA-3';

R: 5'-TTTTCGGCGGATTTTTGC-3'.

atg2: F: 5'-CCAACGCCTATACCATAGTGAGAGA-3'

R: 5'-TCTGGTCGTGCTCCGTGAT-3'

Scanning electron microscopy. Adult flies were anaesthetized to death by chloroform, mounted on stages and then observed using a scanning electron microscopeTM-1000 (HITACHI, Tokyo, Japan).

IOP cell counts. At least 30 hexagonal target areas were scored from three to six different flies for each experiment as reported by others. ${ }^{32}$

\section{Conflict of Interest}

The authors declare no conflict of interest.

Acknowledgements. This work is supported by the Chinese Ministry of Science and Technology (2013CB530700) and National Natural Science Foundation of China (NSFC31171324) to L L.

1. Galluzzi L, Bravo-San Pedro JM, Vitale I, Aaronson SA, Abrams JM, Adam D et al. Essential versus accessory aspects of cell death: recommendations of the NCCD 2015. Cell Death Differ 2015; 22: 58-73.

2. Brachmann CB, Cagan RL. Patterning the fly eye: the role of apoptosis. Trends Genet 2003; 19: $91-96$.

3. Cagan RL, Ready DF. The emergence of order in the Drosophila pupal retina. Dev Biol 1989; 136: 346-362.

4. Hay BA, Wolff T, Rubin GM. Expression of baculovirus P35 prevents cell death in Drosophila. Development 1994; 120: 2121-2129.

5. Copeland JM, Bosdet I, Freeman JD, Guo M, Gorski SM, Hay BA. echinus, required for interommatidial cell sorting and cell death in the Drosophila pupal retina, encodes a protein with homology to ubiquitin-specific proteases. BMC Dev Biol 2007; 7: 82.

6. Lin HV, Rogulja A, Cadigan KM. Wingless eliminates ommatidia from the edge of the developing eye through activation of apoptosis. Development 2004; 131: 2409-2418.

7. Cordero J, Jassim O, Bao S, Cagan R. A role for wingless in an early pupal cell death event that contributes to patterning the Drosophila eye. Mech Dev 2004; 121 : 1523-1530.

8. Miller DT, Cagan RL. Local induction of patterning and programmed cell death in the developing Drosophila retina. Development 1998; 125: 2327-2335.

9. Hay BA, Guo M. Caspase-dependent cell death in Drosophila. Annu Rev Cell Dev Biol 2006; 22: 623-650.

10. Steller H. Regulation of apoptosis in Drosophila. Cell Death Differ 2008; 15: 1132-1138.

11. White K, Grether ME, Abrams JM, Young L, Farrell K, Steller H. Genetic control of programmed cell death in Drosophila. Science 1994; 264: 677-683.

12. Chen $P$, Nordstrom W, Gish B, Abrams JM. grim, a novel cell death gene in Drosophila. Genes Dev 1996; 10: 1773-1782.

13. Grether ME, Abrams JM, Agapite J, White K, Steller H. The head involution defective gene of Drosophila melanogaster functions in programmed cell death. Genes Dev 1995; 9: 1694-1708.

14. Shlevkov E, Morata G. A dp53/JNK-dependant feedback amplification loop is essential for the apoptotic response to stress in Drosophila. Cell Death Differ 2012; 19: 451-460.

15. Brodsky MH, Nordstrom W, Tsang G, Kwan E, Rubin GM, Abrams JM. Drosophila p53 binds a damage response element at the reaper locus. Cell 2000; 101: 103-113.

16. Igaki T, Kanda H, Yamamoto-Goto Y, Kanuka H, Kuranaga E, Aigaki T et al. Eiger, a TNF superfamily ligand that triggers the Drosophila JNK pathway. EMBO J 2002; 21: 3009-3018.

17. Moreno E, Yan M, Basler K. Evolution of TNF signaling mechanisms: JNK-dependent apoptosis triggered by Eiger, the Drosophila homolog of the TNF superfamily. Curr Biol2002; 12: $1263-1268$ 
18. Joza N, Galindo K, Pospisilik JA, Benit P, Rangachari M, Kanitz EE et al. The molecular archaeology of a mitochondrial death effector: AIF in Drosophila. Cell Death Differ 2008; 15: 1009-1018.

19. Berry DL, Baehrecke EH. Growth arrest and autophagy are required for salivary gland cell degradation in Drosophila. Cell 2007; 131: 1137-1148.

20. Denton D, Shravage B, Simin R, Mills K, Berry DL, Baehrecke EH et al. Autophagy, not apoptosis, is essential for midgut cell death in Drosophila. Curr Biol 2009; 19: 1741-1746.

21. Denton D, Nicolson S, Kumar S. Cell death by autophagy: facts and apparent artefacts. Cell Death Differ 2012; 19: 87-95.

22. Fuchs Y, Steller H. Programmed cell death in animal development and disease. Cell 2011; 147: $742-758$.

23. Syntichaki $P$, Tavernarakis $N$. The biochemistry of neuronal necrosis: rogue biology? Nat Rev Neurosci 2003; 4: 672-684.

24. Yuan J, Lipinski M, Degterev A. Diversity in the mechanisms of neuronal cell death. Neuron 2003; 40: 401-413.

25. Crish SD, Calkins DJ. Neurodegeneration in glaucoma: progression and calcium-dependent intracellular mechanisms. Neuroscience 2011; 176: 1-11.

26. Zhivotovsky B, Orrenius S. Calcium and cell death mechanisms: a perspective from the cell death community. Cell Calcium 2011; 50: 211-221.

27. Jenny A. Preparation of adult Drosophila eyes for thin sectioning and microscopic analysis. $J$ Vis Exp 2011; 54: 2959

28. Liu $\mathrm{H}, \mathrm{Ma} \mathrm{C}$, Moses K. Identification and functional characterization of conserved promoter elements from glass: a retinal development gene of Drosophila. Mech Dev 1996; 56: 73-82.

29. Charlton-Perkins M, Cook TA. Building a fly eye: terminal differentiation events of the retina, corneal lens, and pigmented epithelia. Curr Top Dev Biol 2010; 93: 129-173.

30. Ni JQ, Zhou R, Czech B, Liu LP, Holderbaum L, Yang-Zhou D et al. A genome-scale shRNA resource for transgenic RNAi in Drosophila. Nat Methods 2011; 8: 405-407.

31. de Nooij JC, Hariharan IK. Uncoupling cell fate determination from patterned cell division in the Drosophila eye. Science 1995; 270: 983-985.

32. Mendes CS, Arama E, Brown S, Scherr H, Srivastava M, Bergmann A et al. Cytochrome c-d regulates developmental apoptosis in the Drosophila retina. EMBO Rep 2006; 7: 933-939.

33. Johnson RI, Cagan RL. A quantitative method to analyze Drosophila pupal eye patterning PLoS One 2009; 4: e7008.

34. Carrera P, Moshkin YM, Gronke S, Sillje HH, Nigg EA, Jackle H et al. Tousled-like kinase functions with the chromatin assembly pathway regulating nuclear divisions. Genes Dev 2003; 17: 2578-2590.

35. Hendzel MJ, Wei Y, Mancini MA, Van Hooser A, Ranalli T, Brinkley BR et al. Mitosis-specific phosphorylation of histone $\mathrm{H} 3$ initiates primarily within pericentromeric heterochromatin during G2 and spreads in an ordered fashion coincident with mitotic chromosome condensation. Chromosoma 1997; 106: 348-360.
36. Arama E, Steller H. Detection of apoptosis by terminal deoxynucleotidyl transferasemediated dUTP nick-end labeling and acridine orange in Drosophila embryos and adult male gonads. Nat Protoc 2006; 1: 1725-1731.

37. Yang Y, Hou L, Li Y, Ni J, Liu L. Neuronal necrosis and spreading death in a Drosophila genetic model. Cell Death Dis 2013; 4: e723.

38. Bardet PL, Kolahgar G, Mynett A, Miguel-Aliaga I, Briscoe J, Meier P et al. A fluorescent reporter of caspase activity for live imaging. Proc Natl Acad Sci USA 2008; 105 13901-13905.

39. Adachi-Yamada T, Fujimura-Kamada K, Nishida Y, Matsumoto K. Distortion of proximodistal information causes JNK-dependent apoptosis in Drosophila wing. Nature 1999; 400 : 166-169.

40. De Benedetti A. The tousled-like kinases as guardians of genome integrity. ISRN Mol Biol 2012; 2012: 627596.

41. Ren X, Sun J, Housden BE, Hu Y, Roesel C, Lin S et al. Optimized gene editing technology for Drosophila melanogaster using germ line-specific Cas9. Proc Natl Acad Sci USA 2013; 110: 19012-19017.

42. Kohda K, Wang Y, Yuzaki M. Mutation of a glutamate receptor motif reveals its role in gating and delta2 receptor channel properties. Nat Neurosci 2000; 3: 315-322.

43. Han Z, Saam JR, Adams HP, Mango SE, Schumacher JM. The C. elegans Tousled-like kinase (TLK-1) has an essential role in transcription. Curr Biol 2003; 13: 1921-1929.

44. Yu SY, Yoo SJ, Yang L, Zapata C, Srinivasan A, Hay BA et al. A pathway of signals regulating effector and initiator caspases in the developing Drosophila eye. Development 2002; 129 : 3269-3278.

45. Kurada P, White K. Ras promotes cell survival in Drosophila by downregulating hid expression. Cell 1998; 95: 319-329.

46. Bergmann A, Agapite J, McCall K, Steller H. The Drosophila gene hid is a direct molecular target of Ras-dependent survival signaling. Cell 1998; 95: 331-341.

47. Velikkakath AK, Nishimura T, Oita E, Ishihara N, Mizushima N. Mammalian Atg2 proteins are essential for autophagosome formation and important for regulation of size and distribution of lipid droplets. Mol Biol Cell 2012; 23: 896-909.

48. Berridge MJ. Calcium hypothesis of Alzheimer's disease. Pflugers Arch 2010; 459: 441-449.

49. Porter JA, Hicks JL, Williams DS, Montell C. Differential localizations of and requirements for the two Drosophila ninaC kinase/myosins in photoreceptor cells. J Cell Biol 1992; 116 683-693

50. Sweeney ST, Hidalgo A, de Belle JS, Keshishian H. X-gal staining of the central nervous system in adult Drosophila. Cold Spring Harb Protoc 2012; 2012: 239-241.

51. Wu JS, Luo L. A protocol for dissecting Drosophila melanogaster brains for live imaging or immunostaining. Nat Protoc 2006; 1: 2110-2115.

52. Bustin SA, Benes V, Garson JA, Hellemans J, Huggett J, Kubista M et al. The MIQE guidelines: minimum information for publication of quantitative real-time PCR experiments. Clin Chem 2009; 55: 611-622.

Supplementary Information accompanies this paper on Cell Death and Differentiation website (http://www.nature.com/cdd) 